\title{
Modelos de evaluación de la Educación superior: los circuitos de comunicación científica en México
}

\author{
Montserrat García Guerrero ${ }^{1}$ and Carmen Fernández Galán Montemayor ${ }^{2}$
}

\section{Resumen}

Las formas de sociabilidad del conocimiento y los sistemas de evaluación de la Educación superior en México se han bomogeneizado a los estándares internacionales, lo cuales están transitando a circuitos de publicación científica enlazados con métricas y proyectos editoriales que mantienen el control sobre la publicación y sobre la dictaminación de la misma y que abora están influenciados por la tendencia de la Ciencia Abierta. A partir de un análisis de las formas de escritura científica (académica) se propone explorar la articulación de los lineamientos para la formación de investigadores en el nivel superior, con las nuevas políticas de Ciencia Abierta. Los criterios para analizar los modelos de evaluación en México son: producción cientifica, escritura académica, certificaciones, métricas y calidad. La finalidad es comparar el modelo internacional con las prácticas y políticas nacionales. Si bien México busca replicar el modelo internacional, es fundamental incluir otros marcos de referencia, tanto para la evaluación de la Educación superior como sobre los mecanismos de revisión de la calidad científica.

Palabras clave: Publicación cientifica; métricas; evaluación; ciencia abierta

\section{Higher Education Evaluation models: Scientific communication circuits in Mexico}

\begin{abstract}
The sociability of knowledge and the evaluation systems of Higher Education in Mexico have been homogenized to international standards, which are transitioning to scientific publication circuits linked to metrics and editorial projects that maintain control over the publication and the evaluation, that are now influenced by the trend of Open Science. Based on an analysis of the forms of (academic) scientific writing, it is proposed to explore the articulation of the guidelines for the training of researchers at the higher level with the new Open Science policies. The criteria to analyze the evaluation models in Mexico are: scientific production, academic writing, certifications, metrics and quality. The purpose is to compare the international model with national practices and policies. Although Mexico seeks to replicate the international model, it is essential to include other frames of reference, both for the evaluation of higher education and for the mechanisms of scientific quality review.
\end{abstract}

Keywords: Scientific publication; metrics; evaluation; open science

\footnotetext{
${ }^{1}$ Montserrat García Guerrero, Gestora de la Oficina de Ciencia Abierta de la Universidad Autónoma de Zacatecas.

Correo electrónio: montsegarcia@uaz.edu.mx.

${ }^{2}$ Carmen Fernández Galán Montemayor, Docente investigadora de la Universidad Autónoma de Zacatecas, México. Líneas de investigación: Semiótica, Literatura comparada, Filología. Tradiciones herméticas y textos híbridos, fronteras entre literatura y ciencia. Correo electrónico: carmenfgalan@uaz.edu.mx.
} 


\section{Introducción}

La política económica neoliberal ha influenciado las agendas sociales y educativas alrededor del mundo, y el caso de México y en general de la región latinoamericana son claro ejemplo de las influencias de organismos internacionales como el Banco Mundial (BM), la Organización de las Naciones Unidas para la Educación, la Ciencia y la Cultura (UNESCO), la Organización para la Cooperación y el Desarrollo Económicos (OCDE), entre otros. La cuestión del desarrollo de los países latinoamericanos es debatido desde hace décadas por expertos en materia económica y social, quienes han puesto a la educación como impulsora para los países en vías de desarrollo (Arteaga, 2014; Folguera, 2020) a los cuales se condiciona el otorgamiento de préstamos y/o apoyos financieros conforme a la alineación a determinados esquemas educativos como lo apunta Maldonado (2000) para el Banco Mundial, o para la UNESCO que actualmente centra su agenda en los Objetivos de Desarrollo Sostenible (ODS) y una recomendación de Ciencia Abierta en materia educativa (UNESCO, 2019, 2021).

La adición a las prácticas de este tipo de organismos "heredan" o aportan un tipo de prestigio, al ofrecer la utopía de que un país es capaz de entrar a opciones de desarrollo y crecimiento avalados por expertos, reunidos en foros internacionales que promueven la globalización. La realidad es que, en los rankings internacionales de las instituciones mexicanas la Universidad Nacional Autónoma de México (UNAM) ocupa la primera posición del país en ese estándar, y las siguientes instituciones mexicanas mejor posicionadas son privadas (como ITESM e ITAM). El esquema jerárquico se replica a nivel nacional donde se les pide a investigadores de estas instituciones consideradas de renombre (UNAM, ITAM, ITESM, UPN, CIDE, entre otras, la mayoría con sede en la capital del país) que formulen proyectos que después deberán ser recibidos por las demás instituciones involucradas en el quehacer educativo.

Para el presente estudio se emplea una estrategia comparativa y descriptiva de los modelos del Estado de evaluación de la Educación superior en México y sus especificidades contextuales. Se muestra la jerarquía de sistemas y la relación forma-contenido de los modelos internacionales y su adecuación al contexto mexicano. El objetivo es ofrecer una explicación de la disparidad y de la imposibilidad de los países en vías de desarrollo de adecuarse a los estándares internacionales y a la vez atender a las necesidades reales de las regiones.

\section{Evaluación = exámenes internacionales y "credencialización"}

La evaluación de la "calidad" de la Educación superior en México, delineada por el Estado, se ha hecho por dos vías: en primera instancia por exámenes estandarizados a los estudiantes de Educación básica (generalmente delineados a nivel internacional como PISA propuesto por la OCDE) y por el otro, con la medición de los docentes de la Educación por medio de la credencialización, es decir, la obtención de grados y diplomas para el caso de la Educación básica (Latapí, 2004), misma que con la reforma educativa del año 2013 integró la evaluación a través de exámenes a los maestros y que muchos consideran más una reforma laboral que educativa (Trujillo, 2015); para la Educación superior la evaluación es centrada en la productividad docente, como otra forma de credencialización.

La evaluación del docente de la Educación superior por producción académica aparece en la agenda mexicana desde los años 70’s, período donde fueron creados el Consejo Nacional de Ciencia y Tecnología (CONACYT), la Asociación Nacional de Universidades e Instituciones de Educación Superior (ANUIES) y la Ley de Coordinación de la Educación Superior (1978) 
, a los que seguirían varias reformulaciones como plan de acción en los años 80’s y 90’s, época marcada por la entrada de la política neoliberal (López-Noriega y Lagunes Huerta, 2009). Todos esos programas creados durante este período siguen aún vigentes y sólo se han adicionado elementos o modificado algunas de sus directrices, lo que evidencia que el modelo de evaluación y "mejoramiento" educativo no ha cambiado, a lo que se suma la falta de resultados sobre cómo esta medición de la calidad por la credencialización o productividad impacta positivamente en las aulas.

El modelo de cascada se repite a todos los niveles y desde la esfera internacional (que se avala con el discurso de "calidad"), lo que hace que queden fuera del marco de revisión y mejora o adecuaciones para los diferentes contextos en que deben aplicarse. El sistema de evaluación de la Educación superior en México funciona bajo una lógica de evaluación constante utilizando tácticas de estímulo-respuesta para la certificación de la "calidad", lo que en términos sociológicos se califica como violencia o lucha simbólica (Bourdieu, 1988, 1996), donde los mecenazgos de Estado se subordinan a los intereses del capital global. A nivel de educación superior los docentes de instituciones públicas y privadas del país son evaluados con programas como el Sistema Nacional de Investigadores (SNI) del Conacyt, creado en 1984, el Programa de Mejoramiento del Personal Docente (PRODEP) fundado en 1996 y el Programa de Estímulos al Desempeño del Personal Docente (ESDEPED)en 2001, (LópezNoriega y Lagunes Huerta, 2009),y para la SEP, la Unidad del Sistema para la Carrera de las Maestras y los (UCICAMM), antes Carrera Magisterial en 1993, y reformado en el 2019 (SEP, 2019), que promueven la competencia académica y escalafonaria, que ha dejado a un lado aspectos clave de la vinculación con la sociedad, de manera que las Universidades públicas, los Institutos tecnológicos y las Normales están en la dualidad de formar investigadores o técnicos para insertar en el mercado laboral.

La tendencia fue limitar el apoyo financiero a las universidades públicas y promover la apertura de universidades técnicas como es el Colegio Nacional de Educación Profesional Técnica (CONALEP) en 1978, que se abrió con apoyo del Banco Mundial (Maldonado, 2000) y cuya evaluación se concentra en el seguimiento de egresados y su inserción al mercado laboral y, por otro lado, la propuesta de medir la Educación superior con la producción científica de los docentes. Aunque existen otras agencias de evaluación como los Comités Interinstitucionales para la Evaluación de la Educación Superior (CIEES) en 1991, la Federación de Instituciones Particulares de Educación Superior A. C. (FIMPES) en 1992, el Centro Nacional para la Evaluación de la Educación Superior (Ceneval) en 1994, el Consejo para la Acreditación de la Educación Superior (COPAES) en 2000, éstas sólo fungen como certificadoras de procesos educativos y trabajan de forma independiente del Estado que, en algunos de sus organismos oficiales, toma en cuentas estas evaluaciones o certificaciones de calidad (García García y Hervás Torres, 2020).En síntesis, hay dos formas de "credencializar" la Educación superior en México, una por parte de las instituciones privadas, las organizaciones independientes y las normas ISO (Hernández et al. 2013), y otra de los organismos creados por el Estado, motivo de este estudio.

Entre todas las formas de evaluación descritas, sólo Conacyt ofrece un modelo que abarca programas académicos de posgrado en el Padrón Nacional de Posgrados (PNPC) que son certificados a través de pares y de la rendición de cuentas sobre la formación de investigadores, cuyos resultados se miden también en publicaciones, este modelo incluye, como eje central, 


\section{Modelos de evaluación de la Educación superior: los circuitos de comunicación científica en México}

la producción científica tanto de docentes del posgrado como de los alumnos, producción que se mide bajo estándares internacionales..

\section{Sistema de publicaciones periódicas para la producción científica}

Los sistemas de publicación científica son los que sirven de base para la evaluación de la Educación superior en el país, donde se mide la productividad docente tomando como base la cantidad de citas de un texto, lo que representa la calidad del mismo. Existen dos sistemas independientes de medición, Scopus de Elsevier que mide la calidad y citas de las revistas (Cuartiles) y los artículos por separado, y Web Of Science de Thomson Reuters que mide las citas de un artículo y de un investigador con el Factor de Impacto. El resultado es que estas dos empresas, con gran trayectoria (desde el siglo XIX), controlan la medición de los resultados científicos como única alternativa de medida, sobre todo Elsevier que se ha vuelto juez y parte de la evaluación de las publicaciones periódicas a nivel mundial. Este fenómeno está relacionado con la obtención de códigos normalizados o copyright para la edición de libros (ISBN), que era la forma más empleada de publicación de resultados científicos, con un número especifico que identifica una publicación con su editor, de manera que la editorial respalda el discurso y con esto se heredan los atributos o prestigio de la misma al texto. Esta práctica de asignar ISBN a los libros y la extensión de ISSN a las revistas se sigue realizando como control del número de publicaciones en un país, que en México está a cargo de la oficina del Instituto Nacional del Derecho de Autor (INDAUTOR).

La medición de la ciencia por Factor de Impacto y Cuartiles ha hecho que el conocimiento como bien "común" se haya cuestionado en diferentes foros donde surgió la iniciativa del Acceso Abierto (Open Access, con Declaración de Budapest en el año 2002) en un primer momento, y posteriormente de la Ciencia Abierta (Open Science, con una propuesta de la Unión Europea en 2005), como programa de acción en todas las etapas de la investigación para que su acceso abierto impacte en la realidad social y económica. Ya que el Factor de impacto y los Cuartiles se miden por una dinámica de consumidores, en su mayoría del mismo gremio, resulta relevante la búsqueda de otros usuarios. Aunque al inicio las grandes editoriales de que se ha hablado reaccionaron con preocupación ante la Ciencia Abierta, se establecieron Acuerdos Transformadores ${ }^{3}$ y convenios de acción con gobiernos e instituciones que fueron creando esquemas empresariales donde los intereses económicos como empresas editoriales no se vieran afectados. Así surgieron las rutas editoriales para publicaciones periódicas: ruta verde (documento abierto solo para Repositorios), ruta dorada (documento abierto, por incluirse en una publicación abierta), ruta bronce (documentos abiertos solo en las páginas de los editores), ruta híbrida (pago para la apertura del documento por parte del autor) y ruta diamante (documento abierto en todos los niveles para lectura y reproducción) (Universo Abierto, 2019).

Uno de los cambios principales en el modelo de edición antiguo, donde se cobraba al lector por el acceso al documento, fue el cobro al autor por la apertura del mismo, con lo que se lograron mantener los márgenes de beneficio de las editoriales, y como consecuencia aparecieron editoriales depredadoras, que emulan los mecanismos de cobro de las editoriales "reconocidas" y prometen una publicación rápida, aunque no cumplen con los parámetros de revisión por pares que, en teoría, validan la calidad de una investigación y que en la mayoría

\footnotetext{
${ }^{3}$ Como los acuerdos millonarios que viene realizando Elsevier con diferentes instituciones educativas y países para ofrecer opciones de Acceso Abierto para los artículos (Hinchliffe, 2021).
}

Yeiyá 
de los países latinoamericanos se hace de forma gratuita por miembros del gremio, con sueldos que provienen de las instituciones de educación tanto públicas como privadas y con beneficios económicos solo para la editorial.

Otro fenómeno que se ha presentando en fechas recientes es el uso por parte de los investigadores de redes sociales académicas como Academia.edu, Researchgate, entre otras, para compartir sus textos y con esto promover la citación de los mismos y seguir las formas de evaluación que promueven Elsevier y Thomson Reuters, cuando aún no se ha entrado a estos circuitos, situación que se presenta en mayor medida para las Ciencias Sociales y Humanidades. Un requisito del SNI en México es subir un archivo de citación, pero aquellos que no han entrado al sistema global buscan otro tipo de herramientas para dar cumplimiento a esta solicitud, ahí es donde estas redes sociales resultan útiles, junto con Google Scholar y Word Press. Este esquema de publicaciones es otra forma de neocolonización puesto que la evaluación a los investigadores está en empresas trasnacionales que miden el impacto del conocimiento en función de las ganancias editoriales y no del impacto en los usuarios y en la resolución de problemas en los países latinoamericanos.

En lo que se refiere a los géneros discursivos, idiomas y foros en que circula la ciencia las características de los textos son un estándar de lo que se entiende y consume como ciencia. La ciencia actual circula en formato de publicación periódica, con un tipo textual regido por el modelo IMRyD (Introducción, Metodología, Resultados y Discusión), en formato digital, y en circuitos delineados por las editoriales, el idioma internacional es el inglés, y todos los artículos científicos deben incluir un abstract y palabras claves para entrar en los buscadores semánticos de la web. Frente a la estandarización del inglés hay propuestas de crear polycentric plurilingual systems (Piron et al. 2021: 87), que buscan la comunicación de la ciencia de acuerdo a las especificidades de cada región. Para el nivel de Educación superior el docente debe estar familiarizado con la publicación científica y sus géneros discursivos como el pre-print, el paper, el informe y el artículo. Este circuito de comunicación científica está sostenido por los siguientes actores y elementos: los autores, los editores, los evaluadores, las editoriales, los índices y los rankings.

Figura 1. Circuito de comunicación científica internacional como modelo semiótico

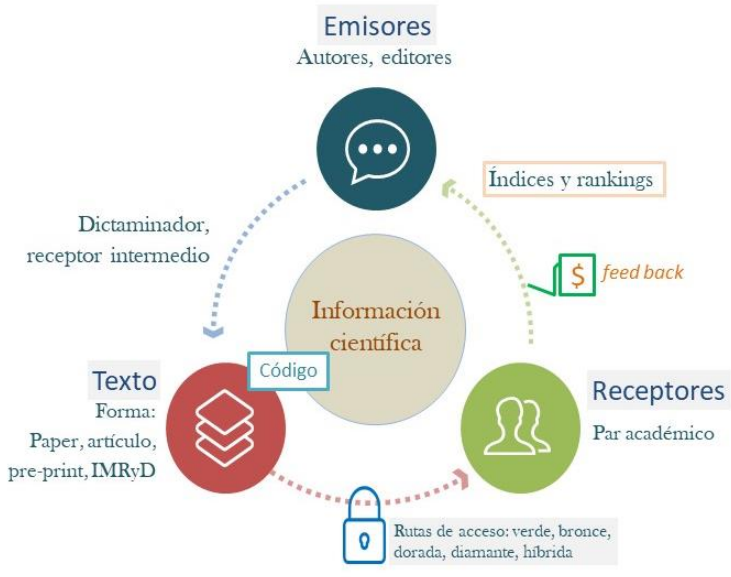

Elaboración propia 
En la fig.1 se muestra cómo la comunicación científica para llegar a su destinatario pasa por varias intermediaciones, el primer elemento es la figura del autor y la propiedad intelectual pues el copyright pasa a los editores, y en el marco de la migración a la digitalización de los saberes se transforma en un identificador alfanumérico y homólogo (ORCID, Researcher ID), en tanto el nombre de la editorial figura como la autentificación del texto. Antes de llegar a su receptor, los textos pasan por un receptor intermedio, los dictaminadores que avalan los contenidos científicos y cuyo arbitraje permanece anónimo (lo que se ha intentado modificar para hacer visible también este proceso de construcción del conocimiento), este dictaminador (es) hace recomendaciones que el autor debe seguir para ajustar su discurso a la forma o codificación de paper, informe, etc. Previo al fin o destinatario último el texto pasa por una segunda codificación al formato de acceso digital establecido para la circulación de la información científica, a saber, las rutas: verde, dorada, diamante e híbrida. En términos semióticos se trata de un modelo de comunicación con multiplicidad de canales e interacción de varios códigos (Klinkenberg, 2006), lo que en su dimensión pragmática fragmenta el proceso comunicativo al cambiar el contexto (Chandler, 2002) de emisión y recepción en la doble codificación del texto, de modo que la forma se impone al contenido, y los resultados de investigación sólo se consumen desde las métricas y no desde la información científica que era la base del mensaje, es decir, los anclajes o referentes del mensaje se vuelven metadiscursivos.

El receptor final de la publicación es, por lo general, otro par académico, en tanto que los emisores (autor y editorial) obtienen un feed back numérico que corresponde a los índices de citación. De esta forma el circuito de comunicación tradicional para el caso de la publicación académica se ve ampliado por intermediarios que controlan el discurso y hacen una traducción a los formatos y circuitos aceptados desde el paradigma vigente que permite que los mensajes puedan ser comunicados. En este esquema la propiedad intelectual de las obras queda en manos de los editores y "los nuevos inquisidores o calificadores son hoy editoriales de «calidad» y organismos de promoción de la ciencia que marcan la tendencia de las prácticas científicas actuales" (García y Fernández Galán, 2021: 34), en una lógica de auto-citación entre las redes académicas.

En el circuito de comunicación del libro impreso descrito por Darnton (2014), los actores son distintos debido al proceso de manufactura, los emisores son igualmente los autores y editores, en tanto los lectores (compradores, bibliotecas, clubes) reciben el texto por la intermediación de proveedores de insumos y mano de obra para los impresores y los encuadernadores que lo entregan a transportistas y vendedores antes de llegar a su consumo. Los efectos sociales de esa experiencia de lectura y recepción del libro son muy diferentes de la lectura fragmentada en los formatos digitales donde el usuario puede editar el texto: "la autoedición significa que cada lector es su propio editor" (Lyons, 2012: 382), llevando a revisitados dilemas de la propiedad intelectual.

\section{Modelos de publicación para la evaluación en México}

La forma en que se mide la Educación superior en México por parte del Estado es por producción científica, retomando el modelo revisado anteriormente, y por tanto influenciada por los intereses de los organismos que las promueven, en vez de ser resultado de un diagnóstico de las necesidades del país y sus regiones. En este sentido es sintomática la 
coincidencia de las fechas de creación de oficinas de Ciencia y Tecnología en varios países de Latinoamérica, pues reflejan las coyunturas de esta política educativa globalizante y neoliberal.

Tabla 1. Oficinas de Ciencia y Tecnología en la región latinoamericana

\begin{tabular}{llrr}
\hline Nombre de oficina & País & Año & Rank \\
\hline $\begin{array}{l}\text { Conselho Nacional de Desenvolvimento Científico } \\
\text { eTecnológico (CNPq) }\end{array}$ & Brasil & 1951 & 1 \\
\hline $\begin{array}{l}\text { Consejo Nacional de Investigaciones Científicas y Técnicas } \\
\text { (CONICET) }\end{array}$ & Argentina & 1958 & 3 \\
\hline $\begin{array}{l}\text { Consejo Nacional de Innovación, Ciencia y Tecnología } \\
\text { (CONICYT) }\end{array}$ & Uruguay & 1961 & 10 \\
\hline $\begin{array}{l}\text { Comisión Nacional de Investigación Científica y Tecnológica } \\
\text { (CONICYT) }\end{array}$ & Chile & 1967 & 4 \\
\hline $\begin{array}{l}\text { Departamento Administrativo de Ciencia, Tecnología e } \\
\text { Innovación (COLCIENCIAS) después Sistema Nacional de }\end{array}$ & Colombia & 1968 & 5 \\
Ciencia, Tecnología e Innovación (SNCTI) en 2019 & & & \\
\hline Consejo Nacional de Ciencia y Tecnología (CONACYT) & México & 1970 & 2 \\
\hline $\begin{array}{l}\text { Ministerio de Ciencia, Tecnología y Telecomunicaciones } \\
\text { (MICIIT) }\end{array}$ & Costa Rica & 1990 & 12 \\
\hline Secretaría Nacional de Ciencia y Tecnología (SENACYT) & Ecuador & 1991 & 9 \\
\hline Consejo Nacional de Ciencia y Tecnología (CONCYT) & Guatemala & 1991 & 17 \\
\hline Consejo Nacional de Ciencia y Tecnología (CONACYT) & El Salvador & 1992 & 25 \\
\hline Ministerio de Ciencia, Tecnología y Medio Ambiente & Cuba & 1994 & 6 \\
\hline $\begin{array}{l}\text { Secretaría Nacional de Ciencia, Tecnología e Innovación } \\
\text { (SENACYT) }\end{array}$ & Panamá & 1997 & 13 \\
\hline $\begin{array}{l}\text { Ministerio de Ciencia y Tecnología (MCT), Sistema Nacional de } \\
\text { Ciencia, Tecnología e Innovación (SNCTI) }\end{array}$ & Venezuela & 1999 & 7 \\
\hline $\begin{array}{l}\text { Consejo Nacional de Ciencia, Tecnología e Innovación } \\
\text { Tecnológica (CONCYTEC) }\end{array}$ & Perú & 2005 & 8 \\
\hline $\begin{array}{l}\text { Elaboración propia con datos de cada oficina y el Scimago Country Rank } \\
\text { Como ser }\end{array}$ & & & \\
\hline
\end{tabular}

Como se puede observar en la tabla 1 la primera oficina de Ciencia y Tecnología creada en América Latina fue la de Brasil y la última, la de Perú, con una diferencia de casi 50 años, ilustrando el hecho de que los diferentes países se han tenido que alinear a la forma impuesta por la agenda neoliberal, México se encuentra a la mitad de la tabla en cuanto a fecha de creación con casi 20 años de diferencia respecto a Brasil, no obstante, estos dos países son los que cuentan con los indicadores de productividad más altos en la región. Cabe destacar que la mayoría de los nombres de las oficinas son similares ya que se pueden contar siete Consejos, tres ministerios, dos Secretarías, un departamento y una Comisión de entre los catorce países presentados en la tabla. Los indicadores de calidad de los rankings son construidos por las editoriales europeas que a partir de sus bases de datos dan informes de citaciones y consultas de los trabajos científicos, y esta es la única opción de medición a nivel mundial. Pero,

¿qué consecuencias trae aparejado comparar una revista británica con una mexicana a partir de sus indices de impacto? ¿Son comparables las citaciones de un autor o autora del Norte con alguno o alguna del Sur? Los métodos cuantitativos de evaluación de la circulación de la ciencia tienden a borrar las diferencias, por tanto, a invisibilizar asimetrías (Salatino y López, 2021: 79).

La ciencia se mide bajo los mismos estándares a nivel mundial, pero no bajo las mismas posibilidades, pues las universidades de los países desarrollados destinan recursos para la 
publicación en revistas de las editoriales distinguidas como autoridad: Springer, Sage, Taylor \& Francis, Emerald, Elsevier, PlosOne, entre otros y en Latinoamérica el pago para el Acceso Abierto se tiene que hacer la mayor parte de las ocasiones a título personal, ya que el apoyo para los cargos por procesamiento de artículos (Article Processing Charges, APC) solo se contempla en algunas instituciones privadas, en resumen instituciones con la capacidad económica y la necesidad de entrar a estos circuitos como estrategia de marketing.

En el Science Ranking de Scimago, que es una de las áreas de acción de la empresa editorial Elsevier, aparece Brasil con más de un millón de documentos, seguido por México con poco más de 300 mil, lo que representa solo una tercera parte del primer lugar de la región y enseguida aparecen Argentina, Chile, Colombia, Cuba, Venezuela, Perú, Ecuador y Uruguay, por nombrar los primeros diez países de ranking. Las tendencias de la investigación globalizada están dirigidas por las políticas de las grandes empresas editoriales que controlan la publicación de los resultados científicos de todo el mundo (Larivière et al. 2015).

El efecto Mateo estudiado por Merton (1968) de que los artículos y autores más citados tienden a seguir siendo muy citados, es un ideal inalcanzable para los investigadores jóvenes de instituciones latinoamericanas por las dificultades para la publicación en las revistas más prestigiosas con la nueva tendencia de rutas de acceso y por la imposibilidad de los países en vías de desarrollo de firmar Acuerdos Transformadores. Con base en las tendencias y programas de evaluación en México, y su relación con las políticas internacionales se presenta un modelo de la forma en que se articulan las tendencias internacionales con las mexicanas.

Figura 2. Modelo de evaluación de la Educación superior en México

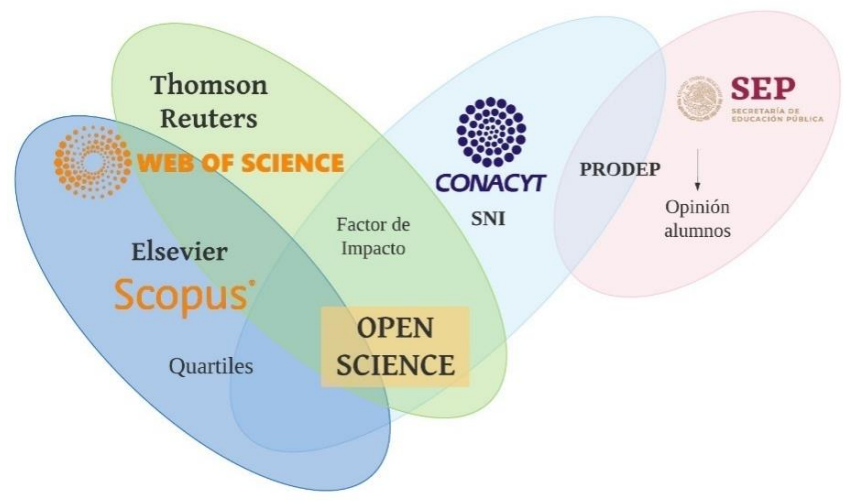

Elaboración propia

La figura 2 muestra la forma en que se relacionan dos formas de medición de la educación superior, la del CONACYT y la SEP (SNI y el PRODEP) con las mediciones de Elsevier y Thomson Reuters (Scopus y Web of Science). El CONACYT está en medio del sistema de evaluación de la SEP (que es el nacional) y de los dos sistemas internacionales de publicación retomando los Cuartiles y el Factor de impacto, como ejes para la rendición de cuentas de los investigadores. La SEP se articula con CONACY'T a través del programa PRODEP que mide además del trabajo frente a grupo, la producción científica, pero no contiene ningún componente de Ciencia Abierta, elemento que sí aparece para Elsevier, Thomson Reuters y CONACYT, sin embargo, la SEP es la única que contiene un esquema de evaluación que contempla a los estudiantes. 
De todos estos actores no hay uno solo que tenga un sistema de medición de otros usuarios del conocimiento, fuera de los pares, no obstante, el esfuerzo reciente del CONACYT por integrar en la evaluación del SNI la divulgación científica en otros circuitos como prensa, radio, TV, e internet (sin especificar los criterios de ponderación de esta producción). El impacto de la ciencia en la sociedad está lejos de poderse medir bajo estos parámetros. Mientras se promueve una evaluación centrada en la productividad para las Universidades, el apoyo de la Secretaría de Educación Pública toma otras rutas: de las 731 Instituciones Públicas de Educación Superior (IES) apoyadas por Prodep en 2020, la mayoría son institutos tecnológicos (370), normales (261) y pocas universidades públicas (35), lo que habla de la respuesta mexicana a las políticas globalizantes de apertura aun mayor número de instituciones de corte tecnológico que representan más de la mitad de las IES públicas (Beech, 2007; Maldonado, 2010).

Las universidades deben de competir con un mercado internacional, donde los certificados respaldan las competencias y los precios de estos diplomas van de acuerdo al prestigio institucional. En el lado de producción académica también se compite con los pares a nivel internacional y la meta es lograr publicar en foros respaldados por grandes corporativos editoriales que controlan el flujo de la ciencia y que se reconocen como el medio legítimo, donde circula el saber científico de actualidad y por tanto donde se está haciendo "verdadera" ciencia. Además de tener las probabilidades numéricas en contra por la cantidad de manuscritos que son rechazados por las editoriales, para lograr la publicación los autores deben seguir ciertas tácticas: "sea interdisciplinario, sea atrevido, o mejor, aparéntelo, sea revisionista, sea impertinente, un poco nada más..." (Darnton, 2014: 108) y, sobre todo, escoger el título adecuado como estrategia de "supervivencia" de los escritores académicos.

A pesar de que uno de los objetivos de la UNESCO es reducir la brecha "digital, tecnológica y de conocimiento" en los países en vías de desarrollo, las recomendaciones del "acceso abierto al conocimiento científico se refiere generalmente al acceso a las publicaciones científicas, los datos de investigación, los programas informáticos, los códigos fuente" (2021:4) y equipos informáticos, es decir, que el concepto de Ciencia Abierta se sustenta en el circuito de comunicación descrito anteriormente que lleva a un autoconsumo y que genera una mayor brecha, obstaculizando la socialización efectiva de los saberes.

\section{Conclusiones}

El discurso de la publicación en editoriales reconocidas como traducción en calidad académica ha impactado en México en dos direcciones, ya que algunos se reconocen en esta manera de entender la ciencia y otros se asumen fuera de este marco de referencia, a lo que se suma la inexistencia de bases de datos que sean lo suficientemente robustas como para ofrecer una medición de la calidad de la publicación y, si bien, que en el marco de la Ciencia Abierta se ha hablado de métricas alternativas, en el año 2021 (enfrentando la pandemia por COVID19) la tendencia sigue siendo publicar en editoriales de "prestigio".

Desde hace algunas décadas, las ciencias, junto con las tecnologías, han sido adoptadas y modificadas desde el poder institucional para incidir de una manera burocrática y publicitaria, centrada en la innovación y en la intensificación del control social, bajo la lógica dominante de la eficiencia (Folguera, 2020: 23).

El modelo exitoso de publicación que es el sueño de los investigadores lo representa la revista Nature de Springer, una de las revistas más importantes a nivel mundial. En las instituciones 


\section{Modelos de evaluación de la Educación superior: los circuitos de comunicación cientifica en México}

se forma a los estudiantes para entender los circuitos de comunicación científica de revistas como ésta que reproduce un esquema de control mutuo (los pares) que prescinde de la vigilancia externa y que perpetúa el ideal del rendimiento. En el gremio académico mexicano se dice que aquel que logra publicar en tales revistas tendrá el reconocimiento como SNI 3 (el nivel más alto) de forma inmediata. A la fecha las evaluaciones de calidad toman en cuenta la publicación en estos monopolios para el otorgamiento de reconocimientos y fondos extraordinarios.

Las instituciones, del mismo modo, terminan siendo medidas con base en estos indicadores de productividad de su planta docente, por lo que el mecanismo regulador se extiende a escala general. Se requiere una adaptación, al menos parcial y la especificidad de las funciones docentes, ya que los investigadores están estratificados socialmente al interior de sus Universidades, sin claridad de las funciones sustantivas para la formación educativa. Mientras no haya alternativas que permitan ponderar la calidad de las obras se sigue dependiendo de lo existente (Scopus y WoS); que tienen la ventaja de promover una comunicación científica eficiente, con propiedad intelectual definida y que en teoría evita malas prácticas: la falsa autoría (poner autores que no hayan participado en el texto), el plagio, el auto plagio (publicación múltiple), la publicación redundante (la paráfrasis de una misma investigación o tema), el efecto salami (varios textos de un solo proceso de investigación fragmentando los resultados y presentando resultados incompletos) y la invención de datos de investigación o tergiversación de los mismos (Del Río et al. 2019; Zúñiga, 2020).

El control y mercantilización de las publicaciones propicia la aparición de editores que ofrecerán maneras más "económicas" de sacar ventaja del sistema, como ocurrió en el siglo XVIII cuando se da la apertura del conocimiento hermético y secreto a las sociedades ilustradas y de acceso abierto a la información, momento histórico en el que los libreros o editores lograron la publicación de obras clandestinas cuyas regalías quedaban fuera del alcance del autor. Entre los procesos de adulteración de libros, Savater destaca este ejemplo: "se tomaba un pedazo de una obra de Voltaire, cuatro de Rousseau y un refrito de no se sabe quién. Precisamente una causa de permanente indignación de Voltaire eran estos procesos de adulteración de sus escritos por causa de la censura" (1997: 26). Los protagonistas actuales de la circulación fuera del marco legal del copyright, son las denominadas web piratas, como SciHub, Lybrary Genesis, The Pirat Date, entre otras. Así como Voltaire se quejaba hace siglos de los editores, ahora los editores se quejan de la piratería cibernética, como Elsevier que demanda a las "webs" por hacer circular los saberes.

Los sistemas de publicación y certificación de la calidad vigentes, han sido fuertemente criticados por promover los monopolios y ostentar el garante de calidad y certificación en los rankings cuando se ha demostrado que estos índices solo miden el número de menciones, aunque estas sean justo para cuestionar la calidad del trabajo. La lucha por la hegemonía del conocimiento se juega en el campo económico en vez del terreno de las ideas y los argumentos. Lo que se lee y se divulga del conocimiento científico está determinado por la forma en que los textos se producen y se distribuyen (Darnton 2003), lo que a su vez marca los patrones de lectura de obras archivadas en hardware y software que se vuelve obsoleto y caduca conforme avanza la tecnología.

El discurso que pone a la educación como el medio para acceder a una mejor calidad de vida lleva al docente de nivel superior en México a la búsqueda no solo de credencializarse sino además de producir a gran escala escritos científicos de calidad. Este docente equivale al

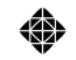

Yeiyá 
cognitariado como un obrero preparado para la nueva etapa económica que pone al conocimiento como el activo principal creando fuerzas productivas para la información (Lozano González y Sicerone, 2018). El desafío es responder a las exigencias de la educación/mercado frente a la carencia de recursos financieros y desde una tradición de escritura científica y circuitos de comunicación diferentes.

Las soluciones a este desafío podrían ser 1) la implementación de ecosistemas tecnológicos para la publicación de calidad (en proceso de construcción), 2) el tránsito hacia métricas alternativas (Altmetrics) para compartir resultados científicos de calidad sin depender del pago de APC's y que respondan adecuadamente a las prioridades de las instituciones. En ese sentido, desde finales de milenio en México se crearon los sistemas Latindex y Redalyc (derivado en AmeliCA) que han evolucionado de la función catalográfica a la indexadora, ofreciendo al país y a la región latinoamericana otros espacios donde se puede hacer visible la producción científica sin depender del circuito y los pagos impuestos desde la agenda internacional, lo que permitirá fomentar nuevos modelos de medición de la calidad educativa a nivel superior y la dirección de los esfuerzos científicos a la solución de problemáticas de la región.

\section{Referencias}

Arteaga Ramírez, L. (2014). "La filosofía y el reto de la educación latinoamericana”. Revista Latinoamericana de Filosofía de la Educación. Volumen 1 - Número 2 - 2014 pp. 175-186.

Bourdieu, P. (1988). "Espacio social y poder simbólico". En: Bourdieu, P. Cosas dichas, Buenos Aires: Gedisa.

Bourdieu, P. y Passeron, J.C. (1996). La Reproducción, Elementos para una teoría del sistema de enseñanza. Madrid: Editorial Popular.

Beech, J. (2007). "La internacionalización de las políticas educativas en América Latina". Rev. Pensamiento Educativo. Vol. 40, n 1, 2007. pp. 153-173.

Darnton, R. (2003). Edición y subversión. Literatura clandestina en el Antiguo Régimen. Vidal trad., TurnerFCE, Madrid.

Darnton. R. (2014). El beso de Lamourette. Reflexiones sobre historia cultural. Saborit trad., FCE, México.

Del Río Martínez, J., \& Del Río Valdés, D. (2019). "Ética y conductas inapropiadas en la práctica de la investigación". Revista de Medicina y Ética, 31(1), 49-69. https://doi.org/https://doi.org/10.36105/mye.2020v31n1.02

Chander, D. (2002). Semiotics. The basis. Routledge, London.

Folguera, Guillermo (2020). La ciencia sin freno. De cómo el poder subordina el conocimiento y transforma nuestras vidas. CFP24 Ediciones.

García García, César, \& Hervás Torres, Mirian. (2020). “Los sistemas de evaluación de la Educación Superior en México y España. Un estudio comparativo". Revista de la educación superior 49(194), 115-136. https://doi.org/10.36857/resu.2020.194.1127

García Guerrero, M. y Fernández Galán Montemayor, C. (2021). "La encrucijada de la Ciencia Abierta: paradigmas y construcción del conocimiento en México". En: Montserrat García Guerrero, Sara María del Patrocinio Rodríguez Palacios, Dagoberto Salas Zendejo, María Soledad Ramírez Montoya y Joel Torres Hernández (coords.), Ciencia Abierta Opciones y experiencias para México y Latinoamérica, Octaedro, Barcelona.

García Peñalvo, F. J., Fidalgo Blanco A. y SeinEchaluce M.L. (2019), "La Presencia del Investigador en el Ecosistema Digital de la Ciencia Abierta". En: M. L. Sein-Echaluce Lacleta, Á. Fidalgo-Blanco, \& F. J. García-Peñalvo (Eds.), Actas del V Congreso Internacional sobre Aprendizaje, Innovación y Competitividad. CINAIC 2019 (9-11 de octubre de 2019, Madrid, España) (pp. 498-503). Zaragoza: Servicio de Publicaciones Universidad de Zaragoza. doi:10.26754/CINAIC.2019.0101 
16 Modelos de evaluación de la Educación superior: los circuitos de comunicación científica en México

Hernández, Gloria, Arcos, José Luis, \& Sevilla, Juan José. (2013). “Gestión de la calidad bajo la Norma ISO 9001”. En: Instituciones públicas de educación superior en México. Calidad en la educación (39), 81 115. https://dx.doi.org/10.4067/S0718-45652013000200004

Hess C. y Ostorm E. (eds) (2016) Los bienes comunes del conocimiento, Traficantes de sueños, Quito, Ecuador.

Hinchliffe, L. J. (2021). The Biggest Big Deal, The Scholarly Kitchen. Disponible en: https://scholarlykitchen.sspnet.org/2021/03/16/the-biggest-big-deal/

Klinkenberg, J. M. (2006). Manual de Semiótica general. Universidad de Bogotá.

Latapí Sarre, Pablo (2004). "La política educativa del Estado mexicano desde 1992” RevistaElectrónica de Investigación Educativa, vol. 6. No. 2. 2004.

Larivière, Vincent, Stefanie Haustein y Philippe Mongeon (2015), “The Oligopoly of Academic Publishers in the Digital Era", PLoS ONE 10(6): e0127502. doi:10.1371/ journal.pone. 0127502

López Noriega M. D. Lagunes Huerta C.A. Recio Urdaneta C.E. (2009). "Políticas públicas y educación superior en México". En: X Congreso Nacional de Investigación Educativa, Veracruz, Veracruz, septiembre de 2009.

Lozano González, M.G. Sicerone D. A. (2018). El cognitariado en el marco de la gubernamentalidad neoliberal. Quorum académico 15(2).

https://produccioncientificaluz.org/index.php/quorum/article/view/29411

Lyons, M. (2012). Historia de la lectura y la escritura en el mundo occidental, Benseñor y Moreno trads., Editoras del Calderón, Buenos Aires.

Maldonado, A. (2000). "Los organismos internacionales y la educación en México. El caso de la educación superior y el Banco Mundial”. Perfiles Educativos, vol. XXII, núm. 87, pp. 51-75.

Merton, Robert K. (1968), “The Matthew Effect in Science”, Science. Vol. 159, No. 3810, pp. 56- 63.

Piron, F., Olyhoek, T., Lujano Vilchis, I., Smith, I. and Liré, Z. (2021). “Saying 'No' to Rankings and Metrics: ScholarlyCommunication and Knowledge Democracy", in: 9789004459076, free access.

https://www.google.com/search?q=Saying $+\% \mathrm{E} 2 \% 80 \% 98 \mathrm{No} \% \mathrm{E} 2 \% 80 \% 99+$ to + Rankings + and + Metrics $\% 3 \mathrm{~A}+$ Scholarly + Communication + and + Knowledge + Democracy\&rlz=1C1CHBF_esMX821MX821\& oq $=$ Saying $+\% \mathrm{E} 2 \% 80 \% 98 \mathrm{No} \% \mathrm{E} 2 \% 80 \% 99+$ to + Rankings + and + Metrics $\% 3 \mathrm{~A}+$ Scholarly + Communica tion + and + Knowledge + Democracy\&aqs $=$ chrome..69i57.1185j0j7\&sourceid $=$ chrome\&ie $=U T F-8$

Rikap, C. y Naidorf, J. (2020). "Ciencia privatizada en América Latina”, Con-Ciencia Social (segunda época) 3, 57-76. DOI:10.7203/con-cienciasocial.3.16790

Salatino, M. y López Ruiz, O. (2021). "El fetichismo de la indexación. Una crítica latinoamericanaa los regímenes de evaluación de la ciencia mundial” Revista CTS, vol. 16, nº 46, 73-100.

Savater, F. (1997). Censura en la Europa ilustrada, en: X. L. Barreiro et al. Censura e Ilustración. Universidad de Santiago de Compostela, 23-35.

Silas-Casillas, J. C. (2021). "Una ley Fifí para la educación superior mexicana". Artículo de opinión. Educación Futura. Disponible en: http://www.educacionfutura.org/una-ley-fifi-para-la-educacionsuperior-mexicana/

Scimago Journal and Country Rank (S/F). Country Ranking. Elsevier. Disponible en: https://www.scimagojr.com/countryrank.php?region=Latin\%20America

Trujillo Holguín, J.A. (2015). "Las reformas educativas en México: un recuento de las modificaciones constitucionales (1934-2013)". En: J.A. Trujillo Holguín, P.

Rubio Molina y J.L. García Leos (coords.), Desarrollo profesional docente: las competencias en el marco de la reforma educativa, Chihuahua, México: Escuela Normal Superior Profr. José E. Medrano R. 77-92.

UNESCO (2019). "Hacia una recomendación de la UNESCO sobre la ciencia abierta." Disponible en: https://en.unesco.org/sites/default/files/open_science_brochure_sp.pdf

UNESCO (2021). "Proyecto de recomendación de la UNESCO sobre la ciencia abierta." Disponible en: https://unesdoc.unesco.org/ark:/48223/pf0000376893_spa?posInSet=1\&queryId=N-EXPLORE8094371a-c1b3-4727-81bb-ba237f38409e

Universo Abierto (2019). "Las 5 rutas para llegar al acceso abierto: verde, dorada, bronce, híbrida y diamante". Disponible en: https://universoabierto.org/2019/03/13/las-5-rutas-para-llegar-al-accesoabierto-verde-dorada-bronce-hibrida-y-diamante/comment-page-1/

Zúñiga, Vargas, J. P. (2020). "Comportamiento ético en la publicación científica: malas conductas y acciones para evitarlas”. Educación, vol. 44, no. 1, 2020, p. NA. Gale Academic OneFile. 